\title{
Hydrophilic interaction liquid chromatography (HILIC) in proteomics
}

\author{
Paul J. Boersema • Shabaz Mohammed • \\ Albert J. R. Heck
}

Received: 23 October 2007 /Revised: 21 December 2007 / Accepted: 9 January 2008 / Published online: 9 February 2008

(C) The Author(s) 2008

\begin{abstract}
In proteomics, nanoflow multidimensional chromatography is now the gold standard for the separation of complex mixtures of peptides as generated by in-solution digestion of whole-cell lysates. Ideally, the different stationary phases used in multidimensional chromatography should provide orthogonal separation characteristics. For this reason, the combination of strong cation exchange chromatography (SCX) and reversed-phase (RP) chromatography is the most widely used combination for the separation of peptides. Here, we review the potential of hydrophilic interaction liquid chromatography (HILIC) as a separation tool in the multidimensional separation of peptides in proteomics applications. Recent work has revealed that HILIC may provide an excellent alternative to SCX, possessing several advantages in the area of separation power and targeted analysis of protein posttranslational modifications.
\end{abstract}

Keywords HILIC $\cdot$ Proteomics $\cdot$ Post-translational modification - Two-dimensional liquid chromatography

\section{Introduction}

Recent years have seen a significant increase of interest in hydrophilic interaction liquid chromatography (HILIC). Although HILIC was first introduced in the 1970s [1], it took until the start of this century for a considerable number

P. J. Boersema $\cdot$ S. Mohammed $\cdot$ A. J. R. Heck $(\square)$

Biomolecular Mass Spectrometry and Proteomics Group,

Bijvoet Center for Biomolecular Research and Utrecht Institute

for Pharmaceutical Sciences, Utrecht University,

Sorbonnelaan 16 ,

3584 CA Utrecht, The Netherlands

e-mail: a.j.r.heck@uu.nl of HILIC applications to appear, highlighting its versatile nature [2-7]. A major cause of this increase is the growing need for the analysis of polar compounds that do not bind to reversed-phase (RP) materials and the constantly increasing complexity of samples [8]. Additionally, the exceptional growth of liquid chromatography-electrospray ionisation-mass spectrometry (LC-ESI-MS) for analytical analyses has led to a widening search for acceptable chromatographic materials. In this search it has been noted that the buffer conditions that are used for HILIC are highly compatible with MS, and that the high organic contents of these buffers can potentially increase sensitivity in ESI-MS $[9,10]$. Moreover, an HILIC separation is "orthogonal" to RP separation, which makes it viable for the multidimensional separation of complex samples [11-13]. Finally, HILIC has been found to allow enrichment and the targeted analysis of post-translational modifications (PTMs) such as glycosylation [14], N-acetylation [12] and phosphorylation [15] in proteomics applications.

Although HILIC applications cover a large range of bioactive compounds, in this review we will focus on the use of HILIC in proteomics. We will describe how HILIC can be used for the separation of peptides in multidimensional chromatography and provide insights into its sensitivity, selectivity, separation power and "orthogonality." We will also discuss how HILIC has recently been used for the enrichment of post-translational modifications at both peptide and protein levels.

\section{HILIC}

HILIC is characterized by the use of a hydrophilic stationary phase and a hydrophobic organic mobile phase [16], which was originally described by Linden et al. in 
1975 [1]. The order of elution is reversed relative to reversed-phase chromatography (RP), with hydrophilic compounds being retained longer than hydrophobic compounds. Therefore, HILIC can simply be seen as a form of normal-phase (NP) chromatography. However, the acronym HILIC was suggested to distinguish it from NP, as NP is typically performed with nonaqueous, non-water-miscible solvent buffers, while HILIC is performed with watermiscible solvents and elution is achieved by a water gradient $[2,9,17,18]$.

In recent years, several stationary phases have emerged that are specifically made for HILIC approaches. Popular phases include underivatized silica stationary phases that contain functional groups such as siloxanes, silanols with (or without) a small quantity of metals $[2,9,19,20]$, derivatized silica, such as the cation exchanger polysulfoethyl A [17, 18], the weak cation exchanger Polycat A [21], the weak anion exchanger PolyWAX [22, 23], TSKgel amide 80 [24, 25], zwitterionic (ZIC)-HILIC [14] and "click" saccharides [26]; see Fig. 1 for structures of typical HILIC phases. Each of these materials display different retention characteristics and separation selectivities and require distinct buffer constitutions for optimal results [10].

A HILIC buffer typically contains more than $70 \%$ acetonitrile [17]. Other eluents have been tested, for instance methanol or isopropanol, but they resulted in poor chromatography or no analyte retention [2]. It is believed that the hydrophilic stationary phase enriches water from the buffer and thus generates an aqueous layer [17]. This allows for hydrophilic analytes to partition between the hydrophilic layer and the hydrophobic elution buffer. Elution is obtained through increasing the hydrophilicity of the mobile phase by increasing the water content. The final separation mechanism of elution, however, is most probably a superpositioning of partitioning and electrostatic interactions or hydrogen bonding to the stationary phase [2]. The extent to which each mechanism dominates is dependent on the actual type of stationary phase used and the buffer conditions, including the level and type of organic content, the type and concentration of salts and the $\mathrm{pH}$ [10]. Charged stationary phases, such as the abovementioned anion or cation exchangers and deprotonated silanol groups, are most likely to display some degree of electrostatic interaction [2]. Buffering salts in the mobile phase can decrease these electrostatic interactions through disruption. The choice of salts is however limited, due to the highly organic conditions of HILIC buffers, which makes it difficult to dissolve some salts in them [10]. Typically, ammonium acetate and formate are chosen because of their compatibility with MS, but ammonium bicarbonate, triethylamine phosphate (TEAP), sodium perchlorate and sodium methylphosphonate $\left(\mathrm{Na}-\mathrm{MePO}_{4}\right)$ have also been found to be applicable [2, 23]. Additionally, the same salt-based disruption can decrease the retention of analytes and can be useful during elution [17]. However, in some instances an increase in retention was seen upon increasing the salt concentration [10]. This was rationalized by the suggestion of a mechanism in which salt is enriched in the aqueous layer, which in
Fig. 1 Chemical structures of the functional groups in common HILIC stationary phases<smiles>CCCNC(=O)CC(CC(=O)NC(CC(=O)NC(CC(=O)NCCS(=O)(=O)O)C(=O)NC(C)(C)C)C(=O)NCCS(=O)(=O)O)C(=O)NCCS(=O)(=O)O</smiles><smiles>CCC[N+](C)(C)CCC[SeH3]</smiles>

ZIC - HILIC<smiles>[R]C(CCCCC(C)C(N)=O)NC([R])([R])C</smiles>

TSKgel Amide-80<smiles>CCCNC(=O)CC(C)(C)C(=O)NC(CC(=O)[O-])C(=O)NC(CC(=O)NC(CC(=O)N[14CH3])C(=O)[O-])C(=O)NCCC</smiles>

PolyCAT A 
turn increases the hydrophilicity of this liquid layer around the stationary phase [10].

Unsurprisingly, the type of salt that is used can also influence the retention behavior. It was observed that basic peptides were retained longer with TEAP in the buffer, while with $\mathrm{Na}-\mathrm{MePO}_{4}$ these were the first peptides to elute with the weak anion exchanger PolyWAX as stationary phase [23]. It was reasoned that TEAP, as a counterion, will generate a sublayer where one negative charge of the salt is attracted to the positively charged stationary phase, while the second negative charge is free to attract basic peptide residues. In the case of $\mathrm{Na}-\mathrm{MePO}_{4}$, there is only one negative charge that interacts with either the stationary phase or basic residues, leaving enough stationary phase available for interaction with acidic peptides [23].

A special type of HILIC called electrostatic repulsionhydrophilic interaction chromatography (ERLIC) actually specifically utilizes the electrostatic interactions in HILIC [23]. In ERLIC, a stationary phase is chosen that has a similar charge to the analytes to be separated. Analytes are on the one hand repelled by the stationary phase but on the other they are retained in the aqueous layer around the stationary phase. These opposing interactions allow isocratic resolution [23]. ERLIC can also be exploited in the selective isolation of phosphopeptides from a tryptic digest $[23,27]$.

Another factor that influences the retention characteristics in HILIC is the $\mathrm{pH}$ of the buffer. Whether the buffer $\mathrm{pH}$ is above or below the $\mathrm{p} K_{\mathrm{a}}$ of the analyte determines its charge state, which in turn affects the hydrophilicity of the analyte and likewise the interaction with the stationary phase $[10,12]$. For example, large differences were found in the retention profiles of a highly complex tryptic digest run over the same column at $\mathrm{pH} 3$ and $\mathrm{pH} 8$ [12]. At $\mathrm{pH} 3$, acidic peptide residues are protonated and so the overall hydrophilicity of peptides containing these residues is decreased, leading to poorer retention of these peptides, with the effect being magnified with higher numbers of acidic residues [12].

For a more detailed overview of different HILIC stationary phases and their applications outside the field of proteomics, see Hëmstrom et al. [2].

\section{HILIC in multidimensional peptide separation}

Shotgun proteomics involves the analysis of entire proteomes in single experiments. Analysis of such samples raises a number of issues, including enormous complexity, large dynamic range and low levels of available analyzable material. Often, SDS PAGE-based [28] or LC separation techniques are used to reduce the sample complexity, followed by RP LC-MS as the final readout. Major advances have been made in the miniaturization of $\mathrm{LC}-$ MS, since reducing the flow rate improves mass spectrometric sensitivity [29], reduces nonspecific adsorption onto separation devices, and improves chromatographic resolving power [30], but improvements are still required. The complexity of a sample can be further reduced prior to mass spectrometric analysis by the addition of extra dimensions of separation. At present, the method of choice is the combination of strong cation exchange (SCX) with RP [31-34]. Over time, a number of alternative configurations have been developed, including size exclusion chromatography (SEC)-RP $[35,36]$ and $\mathrm{RP}$ at $\mathrm{pH} 10$ followed by $\mathrm{RP}$ at $\mathrm{pH} 2.6$ [37]. However, the low separation power of SEC [36] and the correlative separation of RP at different $\mathrm{pH}$ levels has not yielded the optimal two-dimensional (2D) LC system yet.

Recently, HILIC was introduced as a dimension for 2DLC-MS and was shown to remove a few of the disadvantages of existing techniques [11, 12]. Evidently, RP and HILIC buffers are not directly compatible and so online hyphenation of these stationary phases will be difficult. Online hyphenation possesses certain advantages, such as minimal loss of sample, no vial contamination, and no sample dilution [38, 39]. However, performing an experiment in an offline fashion is not necessarily inferior since overloading of the second dimension is no longer an issue with offline fractionation as it is for online approaches. Moreover, in an offline mode, columns and/or buffers that are normally not compatible with the two consecutive separations can be used [38, 40]. Finally, a conventional gradient for peptide separation can be used in offline setups, and this has been shown to be generally superior to step elution [40].

Gilar et al. separated and analyzed a mixture of approximately 200 peptides using different stationary phases, including SEC, HILIC, RP at $\mathrm{pH} 2.6$ and $\mathrm{pH} 10$ and SCX. HILIC was shown to have a separation power superior to both SCX and SEC [11]. The orthogonalityi.e., how different the separation mechanisms are-of the stationary phases was determined by plotting the peptide retention times of two dimensions against each other (see Fig. 2). Although this study did not actually experimentally connect the different stationary phases, since all LC-MS runs were one dimensional, it revealed that HILIC is slightly more orthogonal to RP than SCX [11].

A genuine two-dimensional HILIC-RP system was presented recently [12]. A microliter flow-scale ZIC-HILIC system was connected offline with a nanoliter flow-scale RPLC-MS. For semi-automation, a microliter-scale fraction collector was connected to the HILIC output to mix the eluent with RP- and MS-compatible aqueous buffer and to allow direct consecutive analysis of the HILIC fractions by MS. In this study, a level of orthogonality to RP was found 
Fig. 2 Two-dimensional plots of normalized peptide retention times in HILIC, SCX and RP separation. Both HILIC and SCX have separation mechanisms that are orthogonal to RP, but the clustering of similarly charged peptides in SCX makes this a less optimal first dimension. From the work of Gilar et al. [11] and reproduced with permission from the American Chemical Society

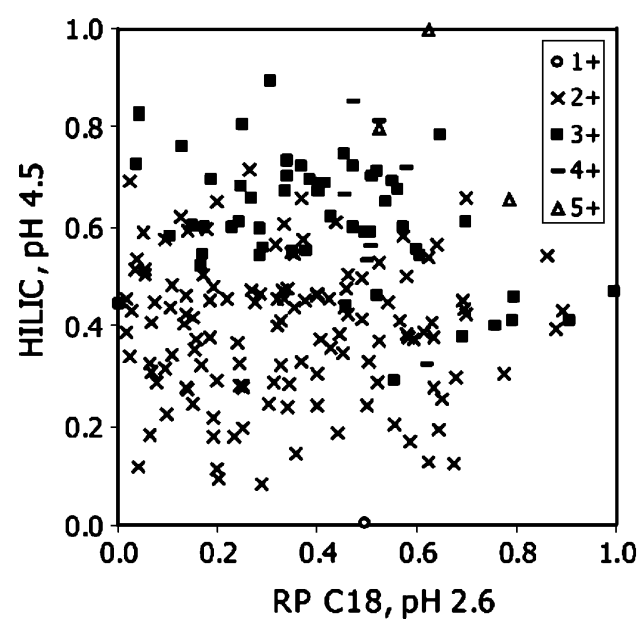

that matched the theoretical picture presented by Gilar et al. [11]. SCX separation can be limited by the fact that similarly charged peptides cluster in narrow elution windows (see also Fig. 2), and it was shown that HILIC did not exhibit this behavior [11, 12].

Analysis of data acquired using this two-dimensional system shed further light on how HILIC can be orthogonal to RP. Indeed, a separation mechanism was observed that is based on both retention by hydrophilicity due to partitioning with the aqueous sublayer and retention by charge due to electrostatic interaction with the stationary phase [11, 12]. These electrostatic interactions ensure that HILIC separation is more than merely the reverse of RP, while the presence of hydrophilic interactions made similarly charged peptides elute over a wider window [12].

It was further noted that the orthogonality of ZIC-HILIC with RP is dependent on the buffer $\mathrm{pH}$. At $\mathrm{pH} 3$, a higher orthogonality was seen than at $\mathrm{pH} 6.8$ and $\mathrm{pH} 8$ [12].

The studies discussed so far were all performed with HILIC columns packed with particles. A further improvement in separation could be obtained by using monolithic structures instead of porous particles. The higher porosity of monolithic materials enables the use of longer columns and/or higher flow rates, increasing the separation efficiency without increasing the backpressure [41]. Recently, attempts to develop HILIC columns that possess monolithic characteristics have been reported [41-45]. Horie et al. demonstrated the use of HILIC monoliths for peptide separations [42]. The authors applied increased flow rates and confirmed that short gradients of 3-10 min were possible with 20-cm-long $200 \mu \mathrm{m}$ ID columns, while the peak capacities were comparable to a similar monolithic RP setup. The poor peptide sequencing speed of a mass spectrometer can be a potential drawback in the direct coupling of such columns to MS. Currently, mass spectrometers can sequence 2-3 peptides per second, which is significantly lower than the number of peptides that will be delivered by the monolith column. However, such columns are highly applicable as a first dimension, where time is not a parameter.

HILIC is proving to be an attractive choice among the range of separation methods available for the proteomics researcher. The combinations chosen so far for 2D-LC-MS, as reported in the literature, have been limited to HILIC$\mathrm{RP}$, but the versatility of the chromatographic material will also allow combinations such as SCX-HILIC and threedimensional approaches can be envisaged. The high organic levels utilized in HILIC buffers compared to RP buffers have the additional benefit that they improve electrospray ionization, which increases the sensitivity of ESI-MS [9], thus suggesting that it may be an interesting final dimension [11].

\section{HILIC for the analysis of PTMs}

The analysis of protein PTMs is a very important albeit challenging task in proteomics. PTMs are generally present at much lower levels than their unmodified counterparts, which makes it very easy to "miss them" in untargeted analyses, such as those performed using shotgun proteomics techniques [46]. Protein modifications such as phosphorylation and glycosylation have proven to be difficult to investigate in a routine fashion. This is mainly because these moieties are prone to elimination and adsorption if care is not taken with sample preparation and MS analysis protocols [14, 47]. Recently, some of these MS problems have been overcome by using alternative MS peptide fragmentation techniques, such as electron capture dissociation (ECD) [48] and electron transfer dissociation (ETD) [49]. Several approaches have been developed to counter some of the problems presented by the low abundance and highly labile nature of PTM peptides. The use of chelation [50-52] or specific chromatographic materials [47] have proven to be successful strategies, and in the latter category HILIC has proven to be a worthy addition. 
HILIC in the targeted analysis of phosphorylated peptides

Phosphorylation is probably the most studied protein posttranslational modification in proteomics; it provides an important function in signal transduction, metabolic maintenance and cell division [53]. The development of techniques to enrich for phosphopeptides has involved major efforts in phosphoproteomics. One of the more successful approaches targets the enrichment of phosphopeptides by SCX at low $\mathrm{pH}$ [47]. At $\mathrm{pH} 3$, acidic residues such as glutamic and aspartic acid are neutral while phospho-serine/threonine/tyrosine will still be negative. Such a distinction allows proteolytic tryptic phosphopeptides to be separated from "normal" tryptic peptides due to an earlier elution. However, other peptide subgroups with reduced net charge, such as $\mathrm{N}$-acetylated tryptic peptides, will also exhibit a shorter retention time, and thus a second level of enrichment is required to separate coeluting nonphosphorylated peptides from phosphorylated peptides [54]. Furthermore, the trypsinization of proteins is less efficient for regions where phosphomoieties are present [55]. Thus a large fraction of proteolytic phosphopeptides will contain miscleavages (i.e., additional basic residues), and will not be enriched. Therefore, other techniques for the targeted enrichment of phosphopeptides are required and are generally performed after SCX, such as those based on immobilized metal affinity chromatography (IMAC) [50, 54] or the metal oxide $\mathrm{TiO}_{2}[51,52,56]$. One other limitation of SCX for phosphopeptide enrichment is that it is based on poor retention. This means that multiply phosphorylated peptides, another large subgroup, are difficult to retain or are even lost. The use of strong anion exchange (SAX) stationary phases immediately following SCX retention can circumvent some of these problems [57]. The generally lower pI values of phosphopeptides will allow stronger retentions on SAX than "normal" peptides. However, the extra LC separation increases the analysis time and the likelihood of sample loss.

Finally, a mixed-bed ion exchange method with both cation and anion exchange was presented recently and was shown to recover more normal tryptic peptides than SCX and to exhibit orthogonal separation towards RP [58]. Phosphopeptides could be eluted by applying a single final salt step. However, in this step only a subset of phosphopeptides - the acidic ones - could be resolved.

HILIC is presented as an alternative enrichment technique, as it exploits the increase in hydrophilicity of peptides through the attachment of a phosphogroup [15, 22, 23, 27]. McNulty et al. applied a TSKgel Amide-80 based HILIC system to the phosphopeptide analysis of a Calyculin A-treated HeLa cell lysate digest. The authors demonstrated that the system, under optimal conditions, allowed phosphorylated peptides to be separated from nonphosphorylated peptides and acidic peptides that might interfere with subsequent IMAC enrichment. Interestingly, the phosphopeptides eluted in the middle of the HILIC separation and would also allow a considerable level of fractionation. This rudimentary analysis permitted more than 1000 unique phosphopeptides to be identified after further IMAC enrichment and LC-MS analysis [15].

Ytterberg et al. evaluated the use of HILIC in a preparative separation set-up using HILIC material packed in micro tips [22]. Different combinations of SCX, RP and two types of HILIC [polyhydroxyethyl aspartamide (PHEA) and PolyWAX] were tested for the enrichment of phosphopeptides from a saliva digest. As expected, most of the phosphorylated peptides were retained strongly under typical HILIC analysis conditions. Furthermore, the separation power of the stepwise elution was sufficient to separate peptides with differing numbers of phosphorylated residues. The conditions used with PolyWAX are ERLIC conditions and seem to be very useful for phosphopeptide enrichment [23]. Normal tryptic peptides are simultaneously repelled by the weak anion exchange material and attracted by hydrophilic interactions, while phosphopeptides are attracted by both. This mode of separation is very responsive to salt contents and the $\mathrm{pH}$ of the buffer and can thus be tweaked to enrich for highly phosphorylated peptides containing up to six phosphorylated residues [22].

In contrast to these results obtained with TSKgel Amide80, PHEA and PolyWAX, the enrichment of phosphopeptides was not observed with ZIC-HILIC [12]. This might be explained by the zwitterionic nature of this chromatographic material: the negative charge could potentially repel the phosphate group, resulting in phosphopeptides eluting with nonphosphorylated peptides.

The microcolumn approach is a simple and undemanding preparative method. However, HPLC-mode HILIC has the potential for automation and fractionation, as the HILIC buffers are directly compatible with IMAC and $\mathrm{TiO}_{2}$. It might be a valuable alternative to SCX, with improved fractionation and better retention of multiply phosphorylated peptides, and so it potentially could allow a more comprehensive analysis of the phosphoproteome. While it should be noted that the application of HILIC to phosphoproteomics is still in its infancy, it is likely that the actual power of HILIC in this field will become clear in the next few years.

HILIC in the targeted analysis of N-terminally acetylated peptides

The positive N-terminal charge of a protein can be neutralized by different post-translational modifications, each potentially influencing protein function, stability and interaction with other biomolecules [59]. Amongst the 
biologically relevant $\mathrm{N}$-terminal modifications are acetylation, methylation and myristoylation [60]. As mentioned above, tryptic $\mathrm{N}$-acetylated peptides can be enriched by SCX [33, 54, 61]. The acetylation neutralizes the Nterminal charge, lowering the net charge compared to the unmodified counterpart. Therefore, $\mathrm{N}$-acetylated tryptic peptides will, like phosphopeptides, elute in the first few SCX fractions.

ZIC-HILIC has been proposed as another means of enriching $\mathrm{N}$-acetylated tryptic peptides [12]. When it contains a neutralized N-terminus, the hydrophilicity of an $\mathrm{N}$-acetylated peptide is decreased. This reduction in polarity is even more pronounced at $\mathrm{pH} 3$, where only basic peptide residues are charged and acidic residues are protonated. Through the use of a ZIC-HILIC separation and fractionation, Boersema et al. showed that this subgroup of trypsinized peptides that are N-terminally blocked could be enriched due to their shorter retentions than "normal" trypsinized peptides (see Fig. 3) [12]. Similarly, other subgroups of N-terminally modified peptides, such as those that contain an $\mathrm{N}$-terminal pyroglutamic acid, were also found primarily in the first ZIC-HILIC fractions [12].

Unfortunately, as trypsin cannot cleave acetylated lysine residues, peptides containing this modification could not be enriched. The miscleavage-containing K-acetylated tryptic peptide will possess a net charge that is the same as "normal" tryptic peptides [12].

Interestingly, and in contrast with SCX [33], no significant enrichment of C-terminal peptides was observed in this ZIC-HILIC set-up, although the hydrophilicity of

pH 3

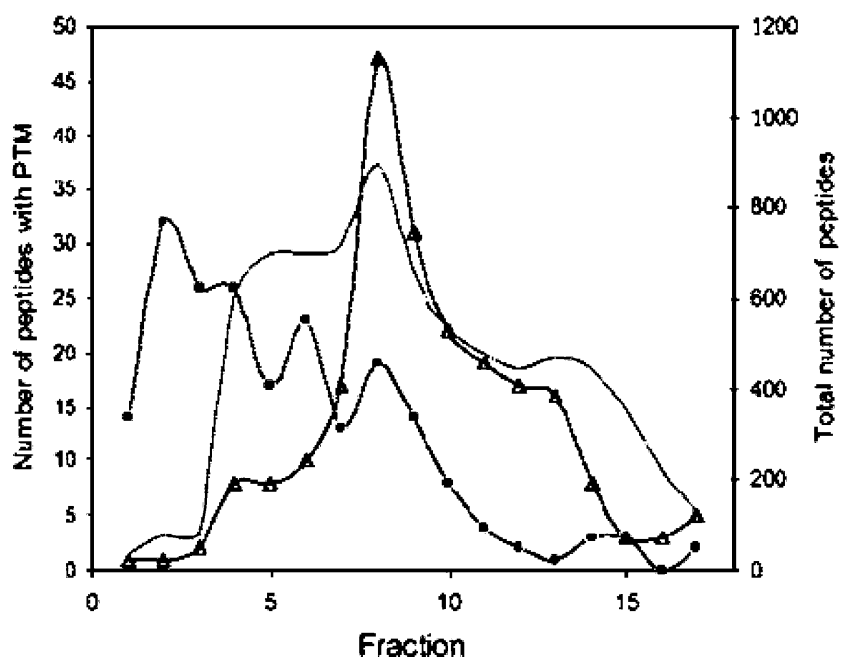

Fig. 3 Distribution of phosphorylated and $\mathrm{N}$-acetylated peptides over ZIC-HILIC fractions. First dimension: ZIC-HILIC, $200 \mu \mathrm{m} \times 160 \mathrm{~mm}$, $3.5 \mu \mathrm{m}, 200 \AA$. Flow rate $1.5 \mu \mathrm{L} / \mathrm{min}, 1 \mathrm{~min}$.fractions. Number of peptides: bare line, total; squares, $\mathrm{N}$-acetylated; triangles, phosphorylated. ZIC-HILIC provides clear enrichment of $N$-acetylated peptides in the initial fractions. From the work of Boersema et al. [12], reproduced with permission from the American Chemical Society these peptides is also different from "normal" tryptic peptides, due to the absence of a terminal arginine or lysine residue. It was hypothesized that this might be explained by the difference in charge distribution after losing an N-terminal positive charge compared to lacking a C-terminal positive charge. A C-terminal peptide would as a result be more polar-and thus more hydrophiliccompared to an N-terminally blocked peptide [12]. As suggested above, the ZIC-HILIC method will enrich for any peptide containing a neutralized $\mathrm{N}$-terminus and thus would be applicable as a first step in the enrichment of formylated, carboxylated, hydroxylated, palmitoylated and myrisotylated peptides.

HILIC in the targeted analysis of glycosylated peptides

Glycosylation, the attachment of a carbohydrate moiety to a protein, is a frequent but very heterogeneous PTM. It modulates the physicochemical and biological properties of proteins and serves as a recognition determinant between molecules, molecules and cells, or between cells [62]. Glycosylation of outer cell wall proteins are, for instance, involved in host-pathogen interactions. It has been estimated that more than half of all proteins in a biological system are glycoproteins [63]. The heterogeneity of glycan structures makes a global proteomics characterization of glycosylation extremely difficult, as the signal intensity of glycosylated peptides with the same amino acid backbone is dispersed over peptides with different oligosaccharide chains [64]. The proteomics analysis of glycopeptides is made even more tedious by the fact that MS/MS data rarely allow the confident identification of peptide sequences due to the relatively large molecular weights of glycosylated peptides [65] and the complex fragmentation that originates from both peptide and glycan cleavages [14]. To overcome some of these issues in proteomics analysis, the labile sugar groups are often removed by deglycosylation prior to MS analysis [66]. The problem of low glycopeptide abundance can be tackled by enrichment techniques, mostly those based on lectin-mediated affinity capture [66]. Recently, HILIC was introduced as a promising additional enrichment step for glycopeptides [14].

The hydrophilicity of glycopeptides makes them ideal candidates for separation by HILIC. A first glycopeptide application for HILIC was found in the analysis of differentially sialylated glycopeptides from interferon- $\gamma$ [67]. Fractions of glycopeptides separated by RP were further separated by HILIC. Their orthogonal retention on the HILIC column was shown to correlate well with the number of sialyl groups. Further studies by ESI-MS revealed that the interaction of N-glycans with ZIC-HILIC is based on a partitioning mechanism, while the separation of differently sialylated $\mathrm{N}$-glycans could be explained by an 
Fig. 4 MALDI-TOF spectra after SPE of a tryptic digest of TIMP-1 by RP (R2, upper spectrum) and HILIC (lower spectrum) microcolumns. HILIC clearly enriches for glycopeptides. This figure was kindly provided by Dr. P. Højrup (similar to [70])

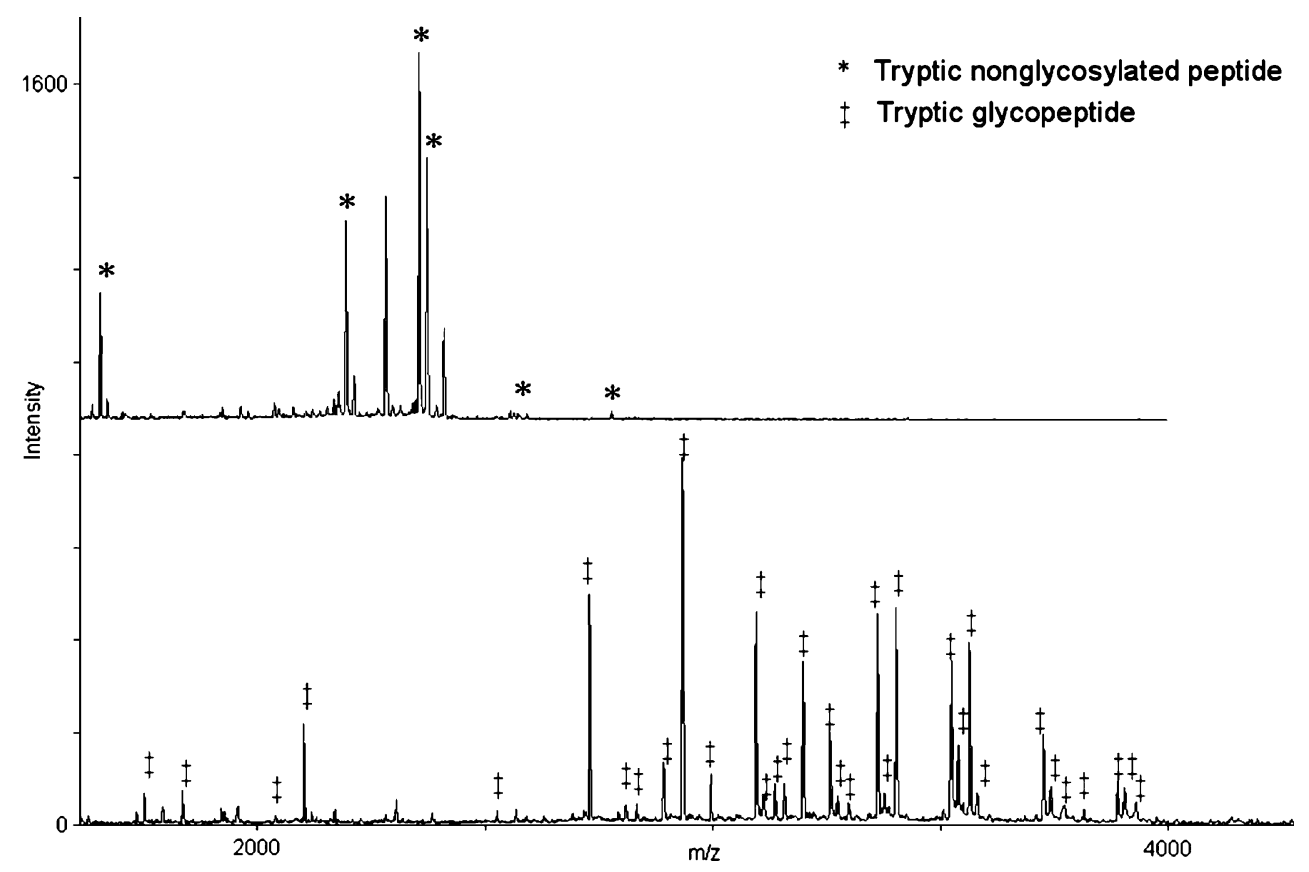

electrostatic repulsion interaction mechanism [68]. Recently, more global proteomics approaches were reported that targeted protein glycosites using HILIC to enrich glycopeptides [14, 66, 69-72]. Generally, in these approaches glycoproteins were first selectively captured by lectinmediated affinity chromatography, followed by SDS-PAGE and in-gel digestion. The peptides obtained were then further enriched for glycosylation using ZIC-HILIC microcolumns. The glycopeptides that were primarily found in the later fractions were then enzymatically degycosylated prior to MS analysis. Figure 4 shows the relative intensities after solid-phase extraction (SPE) of a tryptic digest of TIMP-1 with RP (R2) and HILIC microcolumns. The relative abundance of glycopeptides was significantly increased following enrichment by HILIC. SPE by RP caused the matrix-assisted laser desorption/ionization (MALDI) spectrum to be dominated by nonglycosylated peptides, while, in comparison, SPE with HILIC resulted in a spectrum crowded with glycosylated peptides [70].

Small diversions from this general protocol have been reported and include lectin-mediated affinity capture at the peptide level [69] and the further separation of glycopeptides by SCX [66]. It was shown that the unambiguous localization of glycosylation sites is facilitated by leaving a single GlcNac residue on the site [14] or by tagging the site using ${ }^{18} \mathrm{O}$ isotope labeling [66, 69].

Using the HILIC method, 62 glycosylation sites could be identified for 37 glycoproteins in human plasma [14], while a further study of the Cohn IV fraction of human plasma revealed $103 \mathrm{~N}$-glycosylation sites and 23 fucosylated $\mathrm{N}$ glycosylation sites [66]. $1465 \mathrm{~N}$-glycosylated sites were found on 829 proteins in C. elegans [72]. However, this study was performed using three 50-200 mg samples for affinity chromatography on three different lectin columns, and each LC-MS analysis was performed in triplicate [72], unlike the Cohn IV work, where a $1 \mathrm{mg}$ fraction was used [66].

The glycosylation sites were determined in these studies, but not the actual glycan structure. A few studies have used HILIC for the desalting and enrichment $[65,70]$ or the separation [73] of intact glycopeptides. However, these studies all used relatively simple protein mixtures or even single proteins, indicating that the unambiguous characterization of peptide glycan structures at the proteome level is still rather challenging.

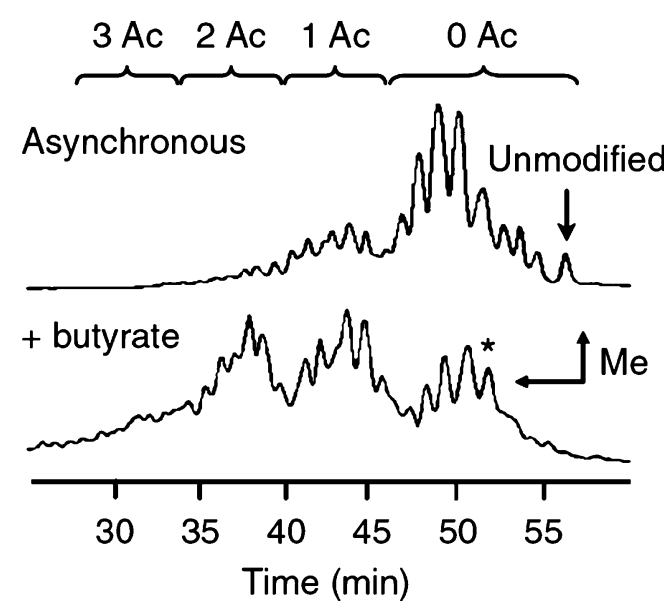

Fig. 5 HILIC separation of GluC-generated long N-terminal peptides from histone H3.2. HILIC primary separation is controlled by the number of acetylations (the three bigger peaks for the +butyrate sample), and within these peaks a secondary separation is observed, relating to the number of methylations (the narrower peaks). Adapted by permission from Macmillan Publishers Ltd: Nature Methods [77] 
These early experiments show that HILIC can be used as an effective and relatively simple tool for the targeted analysis of protein glycosylation. Future studies are likely to follow, wherein HILIC might also have an important role to play in not only glycosite elucidation but also in the compositional analysis of glycan structures, as HILIC was already found to be convenient for the desalting and enrichment [64] or even the separation [74] of glycans themselves.

HILIC in the targeted analysis of histone modifications

The regulation of the chromatin structure, and therefore DNA transcription and replication, is driven by post-translational modifications of the core histones. These modifications, including methylation, acetylation, phosphorylation, sumoylation and ubiquitination, are primarily found at the histone N-terminal tails [75]. Different modifications can occur at the same histone, and it is emerging that not just single site modification but rather the interdependence of these modifications is very important to regulation [76]. The analysis of histone modifications on intact histone proteins by MS is very demanding, due to the enormous heterogeneity of the modifications [77]. The separation of differentially modified proteins prior to MS is therefore essential.

HILIC has been used in the last decade for the analysis of acetylated and methylated histones [5, 7, 21, 78-80]. In these studies, RP and HILIC phases are generally combined to separate different histones. In analyses where LC-MS analysis is used for the characterization of histone modifications, this is performed on the peptide level. However, information on the interdependence of modifications is lost due to the hydrolysis step, because different modifications will end up at different peptides [5, 6, 79-81]. To preserve this information, a HILIC separation of intact histones was recently performed using a top-down MS approach, i.e., at the level of intact proteins [77, 82]. Histone $\mathrm{H} 4$ was purified by RP while HILIC was used for the further separation of differentially modified forms. Subsequently, these H4 HILIC fractions were analyzed by ESI-Q-FTMS/ MS with ECD fragmentation [82]. A similar but semitop-down approach was performed for the analysis of H3.2. As H3.2 is not very soluble in the highly organic HILIC buffers, GluC digestion was employed to generate a 50 amino acid-long N-terminal peptide that contains most of the modification sites [77]. Figure 5 clearly shows the separation power of HILIC in the analysis of histones. It simultaneously separates acetylated and methylated histones, greatly reducing the complexity of the sample. Acetylation is the major determinant of separation. The separation of proteins for which acetylation was induced by butyrate resulted in more peaks at lower retention times. The smaller peaks within these larger peaks represent proteins with different levels of methylation.
In these histone analyses, HILIC delivered the extra separation step that was needed to sufficiently reduce the complexity of the samples and to separate the most important histone modifications.

\section{Concluding remarks}

Recent years have witnessed an increased interest in HILIC. More versatile and diverse stationary phases have become available, leading to reports of an exciting and broad range of applications. The unique separation and orthogonality of HILIC towards RP (the most commonly used peptide separation method) make it an ideal method for multidimensional chromatography that can extend separation power. As far as selectivity is concerned, HILIC can compete well with RP and SCX, which are the two main chromatographic techniques applied today. Focusing solely on proteomics applications, HILIC has been shown to be very versatile in analyses of protein modifications. The retention of hydrophilic compounds can be effectively exploited in the enrichment of phosphorylated, N-terminally blocked and glycosylated peptides. Moreover, in combination with RP it allows the separation of complex differentially modified histones, an incredibly challenging area. It is therefore expected that the number of applications of HILIC in the proteomics field will increase significantly in the years to come, and that the development of novel HILIC stationary phases and/or monolithic columns will continue. Much progress is expected.

Acknowledgements We thank Dr. A.F.M. Altelaar for critically reviewing this manuscript. This work was supported by the Netherlands Proteomics Centre (http://www.netherlandsproteomicscentre.nl).

Open Access This article is distributed under the terms of the Creative Commons Attribution Noncommercial License which permits any noncommercial use, distribution, and reproduction in any medium, provided the original author(s) and source are credited.

\section{References}

1. Linden JC, Lawhead CL (1975) J Chromatogr A 105:125-133

2. Hemström P, Irgum K (2006) J Sep Sci 29:1784-1821

3. Strege MA, Stevenson S, Lawrence SM (2000) Anal Chem 72:4629-4633

4. Risley DS, Strege MA (2000) Anal Chem 72:1736-1739

5. Sarg B, Koutzamani E, Helliger W, Rundquist I, Lindner HH (2002) J Biol Chem 277:39195-39201

6. Sarg B, Helliger W, Hoertnagl B, Puschendorf B, Lindner H (1999) Arch Biochem Biophys 372:333-339

7. Lindner H, Sarg B, Grunicke H, Helliger W (1999) J Cancer Res Clin Oncol 125:182-186

8. Xu RN, Fan L, Rieser MJ, El-Shourbagy TA (2007) J Pharm Biomed Anal 44:342-355

9. Naidong W (2003) J Chromatogr B 796:209-224 
10. Guo Y, Gaiki S (2005) J Chromatogr A 1074:71-80

11. Gilar M, Olivova P, Daly AE, Gebler JC (2005) Anal Chem 77:6426-6434

12. Boersema PJ, Divecha N, Heck AJR, Mohammed S (2007) J Proteome Res 6:937-946

13. Wang XD, Li WY, Rasmussen HT (2005) J Chromatogr A 1083:58-62

14. Hagglund P, Bunkenborg J, Elortza F, Jensen ON, Roepstorff P (2004) J Proteome Res 3:556-566

15. McNulty DE, Annan RS (2007) In: 55th ASMS Conf Mass Spectrometry, Indianapolis, IN, 3-7 June 2007

16. Alpert AJ, Andrews PC (1988) J Chromatogr 443:85-96

17. Alpert AJ (1990) J Chromatogr 499:177-196

18. Zhu B-Y, Mant CT, Hodges RS (1991) J Chromatogr A 548:13-24

19. Nikolov ZL, Reilly PJ (1985) J Chromatogr A 325:287-293

20. Kirkland JJ, Dilks CH, Destefano JJ (1993) J Chromatogr 635:19-30

21. Lindner H, Sarg B, Meraner C, Helliger W (1996) J Chromatogr A 743:137-144

22. Ytterberg JA, Ogorzalek-Loo RR, Boontheung P, Loo JA (2007) In: 55th ASMS Conf Mass Spectrometry, Indianapolis, IN, 3-7 June 2007

23. Alpert AJ (2008) Anal Chem 80:62-76

24. Yoshida T (1997) Anal Chem 69:3038-3043

25. Tomiya N, Awaya J, Kurono M, Endo S, Arata Y, Takahashi N (1988) Anal Biochem 171:73-90

26. Guo Z, Lei A, Zhang Y, Xu Q, Xue X, Zhang F, Liang X (2007) Chem Commun (Camb) 24:2491-2493

27. Alpert AJ, Gygi SP, Shookla AK (2007) In: 55th ASMS Conf Mass Spectrometry, Indianapolis, IN, 3-7 June 2007

28. Rabilloud T (2002) Proteomics 2:3-10

29. Wang H, Hanash S (2003) J Chromatogr B 787:11-18

30. Nagele E, Vollmer M, Horth P, Vad C (2004) Expert Rev Proteomics 1:37-46

31. Dong M-Q, Venable JD, Au N, Xu T, Park SK, Cociorva D, Johnson JR, Dillin A, Yates JR III (2007) Science 317:660-663

32. Brunner E, Ahrens CH, Mohanty S, Baetschmann H, Loevenich S, Potthast F, Deutsch EW, Panse C, de Lichtenberg U, Rinner O, Lee H, Pedrioli PGA, Malmstrom J, Koehler K, Schrimpf S, Krijgsveld J, Kregenow F, Heck AJR, Hafen E, Schlapbach R, Aebersold R (2007) Nat Biotech 25:576-583

33. Dormeyer W, Mohammed S, Breukelen Bv, Krijgsveld J, Heck AJR (2007) J Proteome Res 6:4634-4645

34. Washburn MP, Wolters D, Yates JR (2001) Nat Biotech 19:242-247

35. Lemieux L, Amiot J (1989) J Chromatogr 473:189-206

36. Opiteck GJ, Jorgenson JW, Anderegg RJ (1997) Anal Chem 69:2283-2291

37. Gilar M, Olivova P, Daly AE, Gebler JC (2005) J Sep Sci 28:1694-1703

38. Peng JM, Elias JE, Thoreen CC, Licklider LJ, Gygi SP (2003) J Proteome Res 2:43-50

39. Wagner K, Miliotis T, Marko-Varga G, Bischoff R, Unger KK (2002) Anal Chem 74:809-820

40. Vollmer M, Horth P, Nagele E (2004) Anal Chem 76:5180-5185

41. Pack BW, Risley DS (2005) J Chromatogr A 1073:269-275

42. Horie K, Ikegami T, Hosoya K, Saad N, Fiehn O, Tanaka N (2007) J Chromatogr A 1164:198-205

43. Ikegami T, Fujita H, Horie K, Hosoya K, Tanaka N (2006) Anal Bioanal Chem 386:578-585

44. Ikegami T, Horie K, Jaafar J, Hosoya K, Tanaka N (2007) J Biochem Biophys Methods 70:31-37

45. Ye F, Xie Z, Wong KY (2006) Electrophoresis 27:3373-3380

46. McLachlin DT, Chait BT (2001) Curr Opin Chem Biol 5:591-602

47. Beausoleil SA, Jedrychowski M, Schwartz D, Elias JE, Villen J, Li JX, Cohn MA, Cantley LC, Gygi SP (2004) Proc Natl Acad Sci USA 101:12130-12135

48. Hakansson K, Chalmers MJ, Quinn JP, McFarland MA, Hendrickson CL, Marshall AG (2003) Anal Chem 75:3256-3262

49. Syka JEP, Coon JJ, Schroeder MJ, Shabanowitz J, Hunt DF (2004) Proc Natl Acad Sci USA 101:9528-9533
50. Posewitz MC, Tempst P (1999) Anal Chem 71:2883-2892

51. Pinkse MWH, Uitto PM, Hilhorst MJ, Ooms B, Heck AJR (2004) Anal Chem 76:3935-3943

52. Pinkse MWH, Mohammed S, Gouw JW, van Breukelen B, Vos HR, Heck AJR (2008) J Proteome Res 7:687-697 DOI 10.1021/pr700605z

53. Reinders J, Sickmann A (2005) Proteomics 5:4052-4061

54. Gruhler A, Olsen JV, Mohammed S, Mortensen P, Faergeman NJ, Mann M, Jensen ON (2005) Mol Cell Proteomics 4:310 327

55. Molina H, Horn DM, Tang N, Mathivanan S, Pandey A (2007) Proc Natl Acad Sci USA 104:2199-2204

56. Benschop JJ, Mohammed S, O'Flaherty MC, Heck AJR, Slijper M, Menke FLH (2007) Mol Cell Proteomics 6:1198-1214

57. Dai J, Jin WH, Sheng QH, Shieh CH, Wu JR, Zeng R (2007) J Proteome Res 6:250-262

58. Motoyama A, Xu T, Ruse CI, Wohlschlegel JA, Yates JR (2007) Anal Chem 79:3623-3634

59. Polevoda B, Sherman F (2003) Biochem Biophys Res Commun 308:1-11

60. Polevoda B, Sherman F (2003) J Mol Biol 325:595-622

61. Aivaliotis M, Gevaert K, Falb M, Tebbe A, Konstantinidis K, Bisle B, Klein C, Martens L, Staes A, Timmerman E, VanDamme J, Siedler F, Pfeiffer F, Vandekerckhove J, Oesterhelt D (2007) J Proteome Res 6:2195-2204

62. Lis H, Sharon N (1993) Eur J Biochem 218:1-27

63. Apweiler R, Hermjakob H, Sharon N (1999) Biochim Biophys Acta 1473:4-8

64. Yu YQ, Gilar M, Kaska J, Gebler JC (2005) Rapid Commun Mass Spectrom 19:2331-2336

65. Yu YQ, Fournier J, Gilar M, Gebler JC (2007) Anal Chem 79:1731-1738

66. Hagglund P, Matthiesen R, Elortza F, Hojrup P, Roepstorff P, Jensen ON, Bunkenborg J (2007) J Proteome Res 6:3021-3031

67. Zhang JF, Wang DIC (1998) J Chromatogr B 712:73-82

68. Takegawa Y, Deguchi K, Ito H, Keira T, Nakagawa H, Nishimura S (2006) J Sep Sci 29:2533-2540

69. Kaji H, Yamauchi Y, Takahashi N, Isobe T (2006) Nat Protoc 1:3019-3027

70. Thaysen-Andersen M, Thogersen IB, Nielsen HJ, Lademann U, Brunner N, Enghild JJ, Hojrup P (2007) Mol Cell Proteomics 6:638-647

71. Omaetxebarria MJ, Hagglund P, Elortza F, Hooper NM, Arizmendi JM, Jensen ON (2006) Anal Chem 78:3335-3341

72. Kaji H, Kamiie J-i, Kawakami H, Kido K, Yamauchi Y, Shinkawa T, Taoka M, Takahashi N, Isobe T (2007) Mol Cell Proteomics 6 (12):2100-2109

73. Wuhrer M, Koeleman CAM, Hokke CH, Deelder AM (2005) Anal Chem 77:886-894

74. Zhao J, Qiu W, Simeone DM, Lubman DM (2007) J Proteome Res 6:1126-1138

75. Gilmore JM, Washburn MP (2007) Nat Methods 4:480-481

76. Coon JJ, Ueberheide B, Syka JEP, Dryhurst DD, Ausio J, Shabanowitz J, Hunt DF (2005) Proc Natl Acad Sci USA 102:9463-9468

77. Garcia BA, Pesavento JJ, Mizzen CA, Kelleher NL (2007) Nat Methods 4:487-489

78. Lindner H, Sarg B, Hoertnagl B, Helliger W (1998) J Biol Chem 273:13324-13330

79. Sarg B, Green A, Soderkvist P, Helliger W, Rundquist I, Lindner HH (2005) FEBS J 272:3673-3683

80. Sarg B, Helliger W, Talasz H, Forg B, Lindner HH (2006) J Biol Chem 281:6573-6580

81. Sarg B, Helliger W, Talasz H, Koutzamani E, Lindner HH (2004) J Biol Chem 279:53458-53464

82. Pesavento JJ, Mizzen CA, Kelleher NL (2006) Anal Chem 78:4271-4280 